\title{
Ritz-Regularization versus Least-Square-Regularization. Solution Methods for Integral Equations of the First Kind
}

\section{E. ScHOCK}

Es.werden drei Regularisierungsmethoden diskutiert (Tikhonov-, Ritz- und Galerkin-Methode), und es wird gezeigt, da $\beta$ im Falle positiv definiter oder selbstadjungierter Operatoren die Ritz- und die Galerkiń-Methode (die letzte mit nicht-reellem Parameter) bessere Konvergenzeigenschaften haben als die Tikhonov-Methode.

Обсуждаются три методы регуляризадии (методы Тихонова, Ритца и Галеркина) и показывается, что в случае положительно-определенных или самосопряженных операторов методы Ритца и Галеркина (последннй - с.невещественным параметром) обладают личшим свойством сходимости чем метол Тихонова:

Three principles of regularization methods are discussed (Tikhonov-, Ritz- and Galerkinmethod), and it is shown that in the case of positive definite or of selfadjoint operators the Ritz- and the Galerkin-principles (the latter with non-real parameter) have better properties than the Tikhonov-principle.

\section{Formulation of the minimum principles}

For the (approximate) solution of equations with positive definite operators in a Hilbert space by minimum principles it is common to use a Ritz variational method instead of a least square principle. For linear equations with unbounded operators J. Nitsche [8] has shown that the Ritz method has better convergence properties than.the least square method, for linear equations of the second kind I proved a similar result [9]. For linear equations of the first kind J. N. FrankIn [3] discussed both methods. Here I will improve' the results of $\mathbf{J}$. N. Frankun and will show that also in the case of the finite dimensional approximations the condition numbers of the systems of linear equations which occur in the computational process have better properties in the Ritz case than in the least square case.

In the sequel $H$ is a real infinite dimensional Hilbert space, $W$ is a dense subspace of $H$, and

$$
T: H \rightarrow H, \quad L: W \rightarrow H^{-}
$$

are linear operators, both selfadjoint and positive definite, but $T$ is compact and $L$ has a bounded "(for instance if $L=I, H=W$ ) or a compact (for instance if $L$ is a. differential operator) inverse. For solving equations of the first kind

$$
T x=y
$$

one uses regularization methods to convert the ill-posed problem (1) in a well-posed problem. A. N. TIkHoNov [11] and others considered the positive quadratic functional $Q_{\alpha}: W \rightarrow \mathbf{R}, \alpha>0$, ,

$$
Q_{\alpha}(w)=\|T w-y\|^{2}+\alpha\|L w\|^{2}
$$


and computed the minimum $w_{\mathrm{a}}^{L S}$ of $Q_{\alpha}$

$$
Q_{\alpha}\left(w_{\alpha}{ }^{L S}\right)=\min \left\{Q_{\alpha}(w): w \in W\right\}
$$

or $w_{a, n}^{L S} \in W_{n}$

$$
Q_{\alpha}\left(w_{\alpha, n}^{L S}\right)=\min \left\{Q_{\alpha}(w): w \in W_{n}\right\},
$$

where $W_{n}$ is an $n$-dimensional subspace of $W$. In the sequel $\mathrm{I}$ will call this method the least-square-regularization.

By Ritz-regularization I will denote the following principle: to compute the minimum of the quadratic functional $(\alpha>0)$

$$
R_{\alpha}(w)=(T w, w)-2(\grave{y}, w)+\alpha(L w, \dot{w}) .
$$

'This method is due to M. M. LAdRENT'Ev [5] and A. B. Bakusmrski [1]. Fór sake of simplicity I will always assume that $T$ is injective. Also I will assume that $y \in$ Range $T$. If $\tilde{y}$ is an arbitrary approximation of $y$, than it is known that the least-square-regularization' is not worse than the Ritz-regularization (see e.g. G. VAINikKo [12]).

\section{Ritz-regularization for commuting operators .}

In the case that the operators $T$ and $L$ commute, I can exactly describe the advantage of the Ritz-solution. Let $\left(\tau_{j}\right)$ be the sequence of eigenvalues of $T,\left(\lambda_{i j}\right)$ the sequence of eigenvalues of $L$, and $\left(u_{j}\right)$ the orthonormalized sequence of the eigenvectors both of $\left(\tau_{j}\right)$ and $\left(\lambda_{j}\right)$. The compactness of $T$ implies $0<\tau_{j} \rightarrow 0$, the compactness of $L^{-1}$. implies $0<\lambda_{j} \rightarrow \infty$ or the boundedness of $L^{-1}$ implies $0<\inf \lambda_{j j}$. In each case $\varepsilon_{j}=\frac{\tau_{j}}{\lambda_{j}} \rightarrow 0$.

Let

$$
\hat{x}=\sum \xi_{j} u_{j} .
$$

It is well known, that $w_{\mathrm{a}}^{L S}$ and $w_{\mathrm{a}}{ }^{R}$ have the representations

or

$$
\begin{aligned}
& w_{\alpha}{ }^{L S}=\left(T^{2}+\alpha L^{2}\right)^{-1} T y=\left(T^{2}+\alpha L^{2}\right)^{-1} T^{2} \hat{x}, \\
& w_{\alpha}{ }^{R}=(T+\alpha L)^{-1} y=\left(T^{\prime}+\alpha L\right)^{-1} T \hat{x}
\end{aligned}
$$

$$
w_{a}{ }^{L S}=\sum \frac{\tau_{j}{ }^{2}}{\tau_{j}{ }^{2}+\alpha \lambda_{j}{ }^{2}} \xi_{j} u_{j}, \quad w_{a}{ }^{R}=\sum \frac{\tau_{j}}{\tau_{j}+\alpha \lambda_{j}} \xi_{j} u_{j}
$$

The error norms are

$$
\begin{aligned}
\left\|\hat{x}-w_{\alpha}{ }^{L S}\right\|^{2} & =\sum\left(1-\frac{\tau_{j}^{2}}{\tau_{j}^{2}+\alpha \lambda_{j}{ }^{2}}\right)^{2} \xi_{j}{ }^{2}=\alpha^{2} \sum\left(\frac{\lambda_{j}^{2}}{\tau_{j}{ }^{2}+\dot{\alpha} \lambda_{j}{ }^{2}}\right)^{2} \xi_{j}{ }^{2} \\
= & \alpha^{2} \sum\left(\frac{1}{\dot{\varepsilon}_{j}{ }^{2}+\alpha}\right)^{2} \xi_{j}{ }^{2}
\end{aligned}
$$

and

$$
\begin{aligned}
\left\|\hat{x}=w_{\alpha}^{R}\right\|^{2} & =\sum\left(1-\frac{\tau_{j}}{\tau_{j}+\alpha \lambda_{j}}\right)^{2} \xi_{j}^{2}=\alpha^{2} \sum\left(\frac{\lambda_{j}}{\tau_{j}+\alpha \lambda_{j}}\right)^{2} \xi_{j}^{2} \\
& =\alpha^{2} \sum\left(\frac{1}{\varepsilon_{j}+\alpha}\right)^{2} \xi_{j}{ }^{2} .
\end{aligned}
$$


The linear operator $S_{\alpha}: H \rightarrow H$,

$$
S_{\alpha} x=\sum \frac{\varepsilon_{j}+\alpha}{\varepsilon_{j}^{2}+\alpha}\left(x, u_{j}\right) u_{j}
$$

has the property $\hat{x}-w_{\alpha}{ }^{L S}=S_{\alpha}\left(\hat{x}-w_{a}^{R}\right)$, therefore

$$
\left\|\hat{x}-w_{\alpha}{ }^{L S}\right\| \leqq\left\|S_{\alpha}\right\| \cdot\left\|\hat{x}-w_{\alpha}^{R}\right\|, \quad\left\|\hat{x}-w_{\alpha}^{R}\right\| \leqq\left\|S_{\alpha}{ }^{-1}\right\| \cdot \| \hat{x}-w_{\alpha}{ }^{L} S_{\|} .
$$

Since $S_{\alpha}$ is selfadjoint the norms of $S_{\alpha}$ and $S_{\alpha}^{-1}$ are

$$
\because\left\|S_{\alpha}\right\|=\sup \frac{\varepsilon_{j}+\alpha}{\varepsilon_{j}^{2}+\alpha} \quad \text { and }\left\|S_{\alpha}^{-1}\right\|=\sup \frac{\dot{\varepsilon}^{2}+\alpha}{\varepsilon_{j} \pm \alpha}
$$

The function $f:[0,1] \rightarrow \mathbf{R}$ with $f(\varepsilon)=\frac{\varepsilon+\alpha}{\varepsilon^{2}+\alpha}$ has its maximum for $\varepsilon_{0}=\sqrt{\alpha^{2}+\alpha}-\alpha$ and it has the value $\left\|S_{\alpha}\right\| \leqq f\left(\varepsilon_{0}\right)=\frac{1}{2}\left(\sqrt{\alpha^{2}+\alpha}-\alpha\right)^{-1}=O\left(\alpha^{-1 / 2}\right)$. From max $\frac{1}{f(\varepsilon)}=1$ it follows $\left\|S_{a}^{-1}\right\| \leqq 1$. This proves the following theorem.

Theorem 1: For the errors $\hat{x}-w_{\alpha}^{R}$ and $\hat{x}-w_{\alpha}{ }^{L S}$ hold

$$
\left\|\hat{x}-w^{L S}\right\| \leqq \frac{1}{2}\left(\sqrt{\alpha^{2}+\alpha}-\alpha\right)^{-1}\left\|\hat{x}-w_{\alpha}^{R}\right\|, \quad\left\|\hat{x}-w_{\alpha}^{R}\right\| \leqq\left\|\hat{x}-w_{a}^{L S}\right\| .
$$

Since, $\lim \left\|S_{\alpha}\right\|=\infty$ the Ritz-solution is always better than the least-square: solution. Of course these estimates are the best possible ones. J. N. Frankin [3] compared the minima of the functionals $R_{\alpha}$ and $Q_{\alpha^{2}}$ but since the regularization methods are important for $\lim \alpha=0$ and the numerical problems occur for small $\alpha$, it is adequate to compare $w_{\alpha}{ }^{L S}$ with $w_{\alpha}{ }^{R}$.

The computation of $w_{\alpha}{ }^{R}$ and $w_{\alpha}^{L S}$ are also infinite dimensional problems as the solution of (1), therefore one has to ask for the properties of finite dimensional approximations. The optimal $n$-dimensional approximation of selfadjoint operators is given by approximations which use the eigenfunctions. So if we will compare the optimal least-square solution with the optimal Ritz-solution we have to specialize $W_{n}=\operatorname{span}\left\{u_{1}, u_{2}, \ldots, u_{n}\right\}$.

Corollary 2: In $W_{n}=\operatorname{span}\left\{u_{1}, \ldots, u_{n}\right\}$, for the $n$-dimensional Ritz-solution $w_{a, n}^{R}$ and the n-dimensional least-square-solution $w_{a, n}^{L S}$ hold

$$
\left\|\hat{x}-\dot{w}_{\alpha, n}^{L S}\right\| \leqq \frac{1}{2}\left(\sqrt{\alpha^{2}+\alpha}-\alpha\right)^{-1}\left\|\hat{x}-w_{\alpha, n}^{R}\right\|, \quad\left\|\hat{x}-w_{\alpha, n}^{R}\right\| \leqq\left\|\hat{x}-w_{\alpha, n}^{L S}\right\| .
$$

Proof: Let $P_{n}: H \rightarrow W_{n}$ be the orthogonal projection. Then by the same computation as in the proof of Theorem 2 one gets

$$
\begin{aligned}
& \left\|\hat{x}-\dot{w_{\alpha, n}^{R} \|^{2}}=\dot{\alpha}^{2} \sum_{j=1}^{n}\left(\frac{1}{\varepsilon_{j}+\alpha}\right)^{2} \xi_{j}^{2}+\right\|\left(I-P_{n}\right) \hat{x} \|^{2}, \\
& \left\|\hat{x}-w_{a, n}^{L S}\right\|^{2}=\alpha^{2} \cdot \sum_{j=1}^{n}\left(\frac{1}{\varepsilon_{j}^{2}+\alpha}\right) \xi_{j}{ }^{2}+\left\|\left(I-P_{n}\right) \hat{x}\right\|^{2} .
\end{aligned}
$$


The linear operator $S_{\alpha, n}: H \rightarrow H$,

$$
S_{a, n} u_{j}= \begin{cases}\frac{\varepsilon_{j}+\alpha}{\varepsilon_{j}{ }^{2}+\alpha}, & 1 \leqq j \leqq n \\ u_{j}, & j>n\end{cases}
$$

has the property $\hat{x}-w_{\alpha, n}^{L S}=S_{\alpha, n}\left(\hat{x}-w_{\alpha, n}^{R}\right)$. Since $\left\|S_{\alpha, n}\right\| \leqq\left\|S_{\alpha}\right\| ;\left\|S_{\alpha, n}^{-1}\right\| \leqq 1$, the corollary follows from Theorem 2

\section{The general case}

If $T$ and $L$ do not conmute then the exact behavior of the errors is not so easy, to compute. But it is possible to obtain a result which shows that also in the general case the Ritz-solution behaves better than the least-square-solution.

Theorem 3: For the errors $\hat{x}-w_{\alpha}^{R}$ and $\hat{x}-w_{\alpha}^{L S}$ hold

$$
\hat{x}-w_{\alpha}{ }^{L S}=S_{\alpha}^{\circ}\left(\hat{x}-x_{\alpha}{ }^{R}\right), \quad \hat{x}-x_{\alpha}{ }^{R}=S_{\alpha}{ }^{-1}\left(\hat{x}-\dot{x}_{\alpha}{ }^{L S}\right)
$$

where $S_{\alpha}=I+B_{\alpha} \cdot C$ with $\left\|B_{\alpha}\right\| \geqq \frac{1}{\alpha}$.

Proof: We have again the representations

$$
w_{\alpha}^{L S}=\left(T^{2}+\alpha L^{2}\right)^{-1} T^{2} \hat{x}, \quad w_{\alpha}^{R}=(T+\alpha L)^{-1} T \hat{x} .
$$

The linear operator $S_{\alpha}: H \rightarrow H$ with the property $\hat{x}-w_{a}{ }^{L S}=S_{\alpha}\left(\hat{x}-w_{\alpha}^{R}\right)$ is defined by the equation $I-\left(T^{2}+\alpha L^{2}\right)^{-1} T^{2}=S_{\alpha}\left(I-(T+\alpha L)^{-1} T\right)$. Therefore

$$
\begin{aligned}
S_{\alpha} & =\left(T^{2}+\alpha L^{2}\right)^{-1}\left(L T+\alpha L^{2}\right)=I+\left(\alpha I+L^{-2} T^{2}\right)^{-1}\left(L^{-1} T-L^{-2} T^{2}\right) \\
- & =I+B_{\alpha} \cdot C .
\end{aligned}
$$

The spectrum of $\dot{L}^{-2} T^{2}$ is contained in the non-negative reals, since from $L^{-2} T^{2} v=\lambda v$ follows $T^{2} v=\lambda L^{2} v,\|T v\|^{2}=\lambda .\|L v\|^{2}$, therefore the norm of $B_{a}=\left(\alpha I+L^{-2} T^{2}\right)^{-1}$ is for $\alpha>0$ by $\left[2:\right.$ Cor. $3 /$ p. 566] $\|B\| \geqq \frac{1}{\alpha}$

A similar result is possible for the finite dimensional approximations. Let $W_{n} \subset W$ be an arbitrary $n$-dimensional subspace of $W$ and $P_{n}: H \rightarrow W_{n}$ the orthogonal. projection. Then the minimal solutions of $Q_{a}$ resp. $R_{a}$ in $W_{n}$ have the representations.

$$
\begin{aligned}
& w_{\alpha, n}^{L S}=\left(P_{n} T^{2} P_{n}+\alpha P_{n} \dot{L}^{2} P_{n}\right)^{-1} P_{n} T^{2} \hat{x}, \\
& w_{a, n}^{R}=\left(P_{n} T P_{n}+\alpha P_{n} L P_{n}\right)^{-1} P_{n} T \hat{x} .
\end{aligned}
$$

The operator $S_{a, n}: H \rightarrow H$ with $\hat{x}-w_{a, n}^{L S_{-}}=S_{a, n}\left(\hat{x}-w_{a, n}^{R}\right)$ is defined by

$$
S_{\left.a\right|_{\left(I-P_{n}\right) H}}=I-P_{n} \text {, }
$$

and, using the abbreviations $T_{n} \stackrel{\prime}{=} P_{n} T P_{n}, \quad T_{n}^{2}=P_{n} T^{2} P_{n}, \quad L_{n}=P_{n} L P_{n}$, $L_{n}{ }^{2}=P_{n} L^{2} P_{n}$, for $x \in P_{n} H$

$$
I-\left(T_{n}^{2}+\alpha \dot{L}_{n}^{2}\right)^{-1} T_{n}^{2}=S_{\alpha, n}\left(I-\left(T_{n}+\alpha L_{n}\right)^{-1} T_{n}\right)
$$

Then again

and

$$
S_{a, n}=I+\left(\alpha I+L_{n}{ }^{-2} T_{n}{ }^{2}\right)^{-1} \cdot\left(L_{n}{ }^{-1} T_{n}-L_{n}{ }^{-2} T_{n}{ }^{2}\right)
$$

$$
\left\|B_{\alpha, n}\right\|=\left\|\left(\alpha^{\prime} I+L_{n}{ }^{-2} T_{n}^{2}\right)^{-1}\right\| \geqq \frac{1}{\alpha} .
$$




\section{Galerkin-regularization}

In the case of linear equations of the second kind the Ritz-method leeds to the same equations as the Galerkin-method. But the following example shows that the usual Galerkin method for the equation

$$
T w+\alpha L w=y \text {. }
$$

does not give a convergent set of solutions $w_{\alpha}$ with $\lim _{\alpha \rightarrow 0} w_{\alpha}=\hat{x}$.

Example: Let $T$ be selfadjoint, injective and compact with an infinite number ' of positive and an'infinite number of negative eigenvalues. For $\alpha \neq 0, \alpha \in \mathbf{R}$ the equation

$$
T w+\alpha w \stackrel{=}{=} .
$$

has a solution $w_{\alpha}$, if $-\alpha$ is not an eigenvalue of $T$, so it is nonsense to ask for lim $v_{\alpha}$ also in the general case.

But $I$ will show that there is a convergent regularization method for non-real parameter $\alpha$. For $L=I$ this method is due to A. B. BakUshrnski [1]. Iet $T: H \rightarrow H$ be compact and selfadjoint, $L: W \rightarrow H$ continuous invertible and selfadjoint, $0 \neq \alpha \in \mathbf{R}$. Then $\vec{I}$ call the following method Galerkin-regularization: To compute a solution $w_{i a}^{G} \in W+i W$ resp. $w_{\text {ia,n }}^{G} \in W_{n}+i W_{n}$ from

$$
(T w+i \alpha L w-y, v)=0
$$

for all $v \in W+i \underline{W}$ resp. $v \in W_{n}+i W_{n}$;

In the case of commuting operators $T$ and $L$ as in $\S 2$ one get the solution

$$
w_{i \alpha}^{G}=(T+i \alpha L)^{-1} T \hat{x}=\sum_{j=1}^{\infty} \frac{\tau_{j}}{\tau_{j}+i_{\alpha} \lambda_{j}} \xi_{j} u_{j} .
$$

$r_{\alpha}=\operatorname{Re} w_{i \alpha}^{G}$ and $s_{\alpha}='-\operatorname{Im} w_{i \alpha}^{G}$ have the representations

$$
r_{\alpha}=\sum \frac{\tau_{j}^{2}}{\tau_{j}^{2}+\alpha^{2} \lambda_{j}^{2}} \xi_{j} u_{j}, \quad s_{\alpha}=\alpha \sum \frac{\lambda_{j} \tau_{j}}{\tau_{j}^{2}+\alpha^{2} \lambda_{j j}{ }^{2}} \xi_{j} u_{j} .
$$

This shows

$$
\text { a) } r_{a}=w_{\alpha^{2}}^{L S}, \quad \text { b) } \lim _{\alpha \rightarrow 0} r_{\alpha}=\hat{x}, \quad \text { c) } \lim _{\alpha \rightarrow 0} s_{\alpha}=0 \text {. }
$$

In the general case a similar result holds.

Theorem 4: Let $T: H \rightarrow H, L: W \rightarrow H$ be selfadjoint linear operators, $T$ compact injective, $L$ continuous invertible and $L^{-1}$ compact and positive definit. For real $\alpha \neq 0$ - let $w_{i \mathrm{a}}^{G} \in W+i W$ be the solution of the equation.

$$
\left(T w_{i,}^{G}+i \alpha L w_{i \alpha}^{G}-y, v\right)=0
$$

for all $v \in W+i W$. Then

$$
\begin{aligned}
& \operatorname{Re} w_{i \alpha}^{G}=\left[\left(L^{-1} T\right)^{2}+\alpha^{2} I\right]^{-1}\left(L^{-1} T\right)^{2} \hat{x}, \\
& \lim _{\alpha \rightarrow 0} \operatorname{Re} w_{i \alpha}^{G}=\hat{x}, \quad \lim _{\alpha \rightarrow 0} \operatorname{Im} w_{i \alpha}^{G}=0 .
\end{aligned}
$$

.Proof: Let $r_{\alpha}=\operatorname{Re} w_{i \alpha}^{G}, s_{\alpha}=-\operatorname{Im} w_{i \alpha}^{G}$. Then $T+i \alpha L$ is continuous invertible. Since $L^{-1} T$ is compact. $L^{-1} T+i \alpha I$ is not invertible only in the case if $-i \alpha$ is an , eigenvalue of $L^{-1} T$. But $(T u, u)+i \alpha(L u, u)=0$ contradicts the condition of 
selfadjointness. Thé equation $(T+i \alpha L)\left(r_{\alpha}-i s_{\alpha}\right)=T \hat{x}$ is equivalent to $T r_{\alpha}+\alpha L s_{\alpha}$ $=T \hat{x}, \alpha L r_{\alpha}-T s_{\alpha}=0$. With $r_{\alpha}=\frac{1}{\alpha} L^{-1} T s_{\alpha}$ one get $T L^{-1} T s_{\alpha}+\alpha^{2} L s_{\alpha}=\alpha T \hat{x}$ and

From

$$
\begin{aligned}
& s_{\alpha}=\alpha\left[\left(L^{-1} T\right)^{2}+\alpha^{2} I\right]^{-1}\left(L^{-1} T\right) \hat{x} \\
& r_{\alpha^{*}}=\left[\left(L^{-1} T\right)^{2}+\alpha^{2} I\right]^{-1}\left(L^{-1} T^{\prime}\right)^{2} \hat{x}
\end{aligned}
$$

it follows

$$
\hat{x}-r_{a}=\left(I-\left(L^{-1} T\right)^{2}\left[\left(L^{-1} T\right)^{2}+\alpha^{2} I\right]^{-1}\right) \hat{x}:=\alpha^{2}\left[\left(L^{-1} T\right)^{2}+\alpha^{2} I\right]^{-1} \hat{x}
$$

$$
\left(L^{-1} T\right)^{2}\left(\hat{x}-r_{\alpha}\right)=\alpha^{2} r .
$$

Since $L^{-1}$ is positive definit, it follows

$$
\begin{aligned}
\alpha^{2}\left(L r_{\alpha}, r_{\alpha}\right) & \leqq \alpha^{2}\left(L r_{\alpha}, r_{\alpha}\right)+\left(L^{-1} T\left(\hat{x}-\overline{r_{\alpha}}\right), T\left(\hat{x}-r_{\alpha}\right)\right) \\
& \leqq \alpha^{2}\left(L r_{a} ; r_{\alpha}\right)+\left(L\left(L^{-1} T\right)^{2}\left(\hat{x}-r_{\alpha}\right), \hat{x}-r_{\alpha}\right) \\
& \leqq \alpha^{2}\left(L r_{\alpha}, r_{\alpha}\right)+\alpha^{2}\left(L r_{\alpha}, \hat{x}-r_{\alpha}\right) \leqq \alpha^{2}\left(L r_{\alpha}, \hat{x}\right)
\end{aligned}
$$

Therefore for all $\alpha>0$

$$
w_{\alpha} \in K:=\{w \in W:(L w, w) \leqq(L w, \hat{x})\} .
$$

But $K$ is contained in the relativ compact set $\{w \in W:(L w, w) \leqq(L \hat{x}, \hat{x})\}$ because $w \in K$ implies $\left\|L^{1 / 2} w\right\|^{2} \leqq\left(L^{1 / 2} w, L^{1 / 2} \hat{x}\right) \leqq\left\|L^{1 / 2} w\right\| \cdot\left\|L^{1 / 2} \hat{x}\right\|$. The injective mapping $\left.\left(L^{-1} T\right)^{2}\right|_{\bar{K}}: \bar{K} \rightarrow \overline{\left(L^{-1} T\right)^{2} K}$ is continuous invertible, therefore (3) implies lini $r_{\alpha}=\hat{x}$ and therefore $\lim s_{\alpha}=0$

The representation

$$
w_{a^{2}}^{L S}=\left[L^{-2} T^{2}+\alpha^{2} I\right]^{-1} L^{-2} T^{2} \hat{x}
$$

shows that $r_{\alpha}$ differs from $w_{a^{2}}^{L S}$ only by a quantity which is given by the measure of non-commutativity of $L$ and $T$.

C. W. Groetsch [4] discusses the convergence of the finite dimensional approximations $w_{a, n}^{L S}$ in the case $L=I$. In this case $r_{\alpha, n}=w_{\alpha^{2}, n}^{L S}$, so his convergence properties also hold in the case of finite dimensional Galerkin-approximations.

\section{Condition numbers}

The approximate solutions $w_{a, n}^{L S}, w_{a, n}^{R} \in W_{n}$ resp. $w_{i a, n}^{G} \in W_{n}+i W_{n}$ of the leastsquare-regularization, Ritz-regularization resp. Galerkin-regularization are determined by systems of linear equations. Here I will discuss the conditions of the systems. The condition of a matrix $A$ is defined by

$$
x=\|A\|:\left\|A^{-1}\right\| \text {. }
$$

In the case of Hilbert space operators it is adequate to use the spectral norms of the matrices.

For arbitrary subspaces $W_{n}$ and non-commuting operators $T$ and $L$ it seems to be impossible to find a satisfactory estimate of the conditions. But it is easy to com-. pute the conditions in the case of optimal choice of $W_{n}$ for commuting operators and to compare these optimal conditions.

The following general lenıma is folklore. 
Lemma'5: Let $S: H \rightarrow$ H be a selfadjoint compact positive definite linear operator. Let $\left(\mu_{n}\right)$ be the monotone ordered sequence of eigenvalues of $S$. Let $\hat{P}_{n}$ be the orthogonal projection onto the space of the first $n$ eigenvectors and $P_{n}$ an orthogonal projection onto an arbitrary $n$-dimensional subspace with

$$
\|S\|=\left\|\hat{P}_{n} S P_{n}\right\|=\left\|P_{n} S P_{n}\right\| \text {. }
$$

Then the optimal condition of the projected operator is

$$
\varkappa_{\mathrm{opt}}=\varkappa\left(\hat{P}_{n} S \hat{P}_{n}\right)=\frac{\mu_{1}}{\mu_{n}} \leqq \varkappa\left(P_{n} S P_{n}\right) .
$$

Proof: The extremal principle of Poincaré and Fischer states

$$
\mu_{n}^{\prime}=\max _{H_{n} \subset H} \min _{x \in H_{n}} \frac{(S x, x)}{(x, x)} \geqq \min _{x \in P_{n} H} \frac{(S x ; x)}{(x, x)}=\min _{x \in P_{n} H} \frac{\left(P_{n} S P_{n} x, x\right)}{(x, x)}=\mu_{n}\left(P_{n} S P_{n}\right)
$$

To compute the conditions with respect to the solution $\hat{x}$ I assume the following assumptions and normalizations: Let $\dot{T}$ and $L$ commute, the quotients $\varepsilon_{j}=\frac{\tau_{j}}{\lambda_{j}}$
monotone, $\varepsilon_{\mathrm{t}}=1, W_{n} \subset H$ with $u_{1} \in W_{n}$. Then the following theorem is true.

Theorem 6: For positive definite operators $L$ and $T$ the condition $\varkappa\left(R_{a, n}\right)$ of the Ritz-system

is

$$
\sum_{j=1}^{n} \zeta_{j}\left[\left(T w_{j}, w_{k}\right)^{\prime}+\alpha\left(L w_{i}, w_{k}\right)\right]=\cdot\left(\hat{x}, T w_{k}\right), \quad k=1,2, \ldots, n
$$

$$
\varkappa\left(R_{\alpha, n}\right) \geqq \chi_{\mathrm{opt}}\left(R_{\alpha, n}\right)=\frac{1+\alpha \varepsilon_{n}^{-i}}{1+\alpha},
$$

'the condition $x\left(L S_{a, n}\right)$ of the least-square-system

$$
\sum_{j=1}^{n} \zeta_{j}\left[\left(T w_{j}, T w_{k}\right)+\alpha\left(L w_{j}, L w_{k}\right)\right]=\left(\hat{x}, T^{2} w_{k}\right), \quad k \doteq 1,2, \ldots, n
$$

is

$$
\varkappa\left(L S_{a, n}\right) \geqq \varkappa_{0 p t}\left(L S_{a, n}\right)=\frac{1+\alpha \varepsilon_{n}^{-2}}{1+\alpha},
$$

and the condition $\varkappa\left(G_{\alpha, n}\right)$ of the Galerkin-system

is

$$
\sum_{j=1}^{n} \zeta_{j}\left[\left(T \hat{w}_{j}, w_{k}\right)+i \alpha\left(L w_{j}, w_{k}\right)\right]=\left(\hat{x}, T w_{k}\right), \quad k=1,2, \ldots, n
$$

$$
x\left(G_{\alpha, n}\right) \geqq x_{\mathrm{opt}}\left(G_{\alpha, n}\right)=\sqrt{\frac{1+\alpha^{2} \varepsilon_{n}^{-2}}{.1+\alpha^{2}}} .
$$

Proof: By Lemma 5 the optimal condition is obtained by using the first $n$ eigenfunctions, so the systems of linear equations have diagonal matrices with the elements

$$
\left(1+\alpha \varepsilon_{j}^{-1}\right) \delta_{j k}, \quad\left(1+\alpha^{2} \varepsilon_{j}^{-2}\right) \delta_{j k}, \quad\left(1+i \alpha \varepsilon_{j}^{-1}\right) \delta_{j k}
$$

for the Ritz-, least-square- resp. Galerkin-system. So the modules of the eigenvalues are obvious

I remark that it follows

$$
\varkappa_{\mathrm{opt}}\left(G_{a, n}\right)=\sqrt{\varkappa_{\mathrm{opt}}\left(L S_{\alpha^{2}, n}\right)} \approx \grave{\varkappa}_{\mathrm{opt}}\left(R_{\alpha, n}\right) .
$$

This shows again the advantage of the Ritz- and the Galerkin-regularization. 


\section{REFERENCES}

[1] БАкушинскиЙ, А. Б.: Об одном численном методе решения интегральных уравнений Фредгольма I рода. ЖК. вычисл. мат. и мат. физ. 5 (1965), 744-7499.

[2] Dunford, N., and J. T. Schwartz: Linear Operators. I: General Theory. New YorkLondon: Interscience Publ. 1958.

[3] Frankin, J. N.: Minimum Principles for Ill-Posed Problems. SIAM J. Math. 'Anal. 9 (1978), 638-650.

[4] Groetsch, C. W.: On a Regularization-Ritz-Method for Fredholm Equations of the First

. Kind. J. Integral Equations 4.(1982), 173-182.

[5] ЛАвРе́Итьв, М. М.: О некоторых некорректных задачах математической фйиики. Новосибирск: Изд-во Сиб. отд-ия Акад. Наук СССР 1962.

[6] LUCas, M. A.: Regularization. In: The Application and Numerical Solution of Integral Equations (Eds.: R. A. Anderssen, F. R. de Hoog, M. A. Lucas). Amsterdam: Noordhoff 1980 .

[7] Marti, J. T.: On the Convergence of an Algorithm Computing Minimum-Norm Solutions of Ill-Posed Problems. Math. Comp. 34 (1980), 521-527.

[8] Nirsche, J.: Zur Konvcrgenz des Ritz'schen Verfuhrens und der Fehlerquadratmethode. In: Numerische Mathematik, Differentialgleichungen, Approximationstheorie (Eds.: L. Collatz, G. Meinardus, H. Unger). Basel: Birkhäuser-Verlag 1968.

[9] 'Scноск, E.: Modifizierte Ritz ${ }_{\mathrm{i}}$ Verfahren. Computing 7 (1971), 46-52.'

[10] StoER, J.: Einführung in die Numerische Mathematik I. Berlin-Heidelberg-New York: Springer-Verlag 1972.

[11] Тихонов, А. Н.: О регуляризации некорректно поставленных задач. Докл. Акад. . , Наук CCCP 153 (1963), 49-52.

[12] Вайникко, Г.: Методы решения линейных некорректно поставленных задач. Тарту (СССР): Тартускй У.н-т 1982.

Manuskripteingáng: 15.07. 1983; in revidierter ,Fassung: 30.05. 1984

\section{VERFASSER:}

$\lambda$ Prof. Dr. Eberhard Schock

Fachbereich Mathematik der Universität

D.6750 Kaiserslautern, Erwin-Schrödinger-Str., PF 3049 